\title{
Scalable Spatiotemporal Crowdsourcing for Smart Cities based on Particle Filtering
}

\author{
Wei Li \\ Dept. of Computer Science and Software Engineering \\ Auburn University, Auburn, AL 36849, USA \\ wei.li@auburn.edu \\ Wei-Shinn $\mathrm{Ku}$ \\ Dept. of Computer Science and Software Engineering \\ Auburn University, Auburn, AL 36849, USA \\ weishinn@auburn.edu
}

\begin{abstract}
In mobile crowdsourcing, workers are financially motivated to perform as many self-selected tasks as possible to maximize their revenue. Unfortunately, the existing task scheduling approaches in mobile crowdsourcing fail to consider task execution duration and do not scale for massive tasks and large geographic areas (e.g., a whole city). In this paper, we study on the geo-task scheduling problem (GTS) under the various spatial and temporal constraints in real-world mobile crowdsourcing applications, including task execution duration and task expiration time. Given the location of a worker, the goal of our study is to find an optimal task execution sequence that maximizes the number of tasks that could be finished. Since the exact solution to the maximum task scheduling is computationally intractable, we propose two sub-optimal approaches (LCPF and NUD-IC) based on the particle filtering and the DBSCAN clustering.
\end{abstract}

\section{KEYWORDS}

Mobile Crowdsourcing, Particle Filtering, DBSCAN

\section{INTRODUCTION}

In mobile crowdsourcing, obtaining an optimal or near-optimal task schedule for a worker to accomplish as many tasks as possible is a crucial yet quite challenging problem. However, the existing task scheduling solutions [2] [7] simply ignore the task execution duration and treat the task expiration time as the only temporal factor in task scheduling, which may lead to very poor results. Take the scenario shown in Figure 1 as an example. A worker at location $w$ has five tasks to complete, $s_{1}, s_{2}, s_{3}, s_{4}$ and $s_{5}$, each with a preset expiration time. According to the existing scheduling solutions [2] [7], $s_{1}$ will be selected as the first option to execute because it is geographically closest to $w$. Considering that $s_{1}$ needs one hour to finish while all the other four tasks only need one minute to complete, it is very likely that $s_{2}, s_{3}, s_{4}$, and $s_{5}$ will all expire after

Permission to make digital or hard copies of part or all of this work for personal or classroom use is granted without fee provided that copies are not made or distributed for profit or commercial advantage and that copies bear this notice and the full citation on the first page. Copyrights for third-party components of this work must be honored.

For all other uses, contact the owner/author(s).

SIGSPATIAL'17, Los Angeles Area, CA, USA

() 2017 Copyright held by the owner/author(s). 978-1-4503-5490-5/17/11 . \$ $\$ 15.00$ DOI: $10.1145 / 3139958.3139973$

\author{
Haiquan Chen \\ Department of Computer Science \\ California State University, Sacramento, CA 95819, USA \\ haiquan.chen@csus.edu \\ Xiao Qin \\ Dept. of Computer Science and Software Engineering \\ Auburn University, Auburn, AL 36849, USA \\ xqin@auburn.edu
}

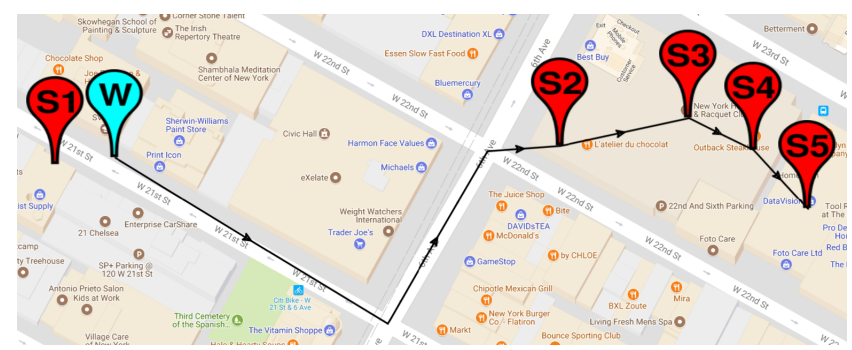

Figure 1: An example of spatial task scheduling in Manhattan island, New York City.

finishing $s_{1}$, leading to the fact that the worker can only accomplish one task. However, the optimal solution in the case should be the task sequence $s_{2} \rightarrow s_{3} \rightarrow s_{4} \rightarrow s_{5}$ so that the worker can have a higher probability to get more tasks completed. In this paper, we study on the geo-task scheduling (GTS) problem in support of realworld mobile crowdsourcing applications that preforms scheduling by taking into account various spatial and temporal constraints, including task execution duration, task expiration time, and task geographic locations. Our study is motivated by the following emerging challenges in mobile crowdsourcing applications: (1) scalability issues due to the growing popularity, (2) scheduling under various spatial and temporal constraints, and (3) incorporating task execution duration into spatial task scheduling.

Table 1: Performance of the Brute Force Task Scheduling

\begin{tabular}{|c|c|c|c|c|c|}
\hline No. of Total Tasks & 20 & 25 & 30 & 35 & 40 \\
\hline Response Time & 24 secs & 3 mins & 22 mins & 67 mins & $>1$ day \\
\hline No. of Tasks Completed & 13 & 14 & 16 & 17 & N/A \\
\hline
\end{tabular}

Scalability issues in mobile crowdsourcing: The ubiquity of mobile platforms and smart phones breeds a large number of mobile crowdsourcing applications like TaskRabbit (a mobile marketplace allowing users to outsource small tasks to workers in their neighborhood [1]), Uber (a mobile application allowing passengers with smartphones to submit trip requests which are then routed to willing drivers [6]), Gigwalk (a crowdsourcing service that helps businesses to appraise their performance [5]), and MediaQ (an online media management framework allowing workers to collect, organize, share, search, and trade user-generated mobile images and videos [8]). A common feature of all these applications is that workers are required to go to the exact spot of each task in person and perform the task by means of a mobile device. Typically a worker has more than one task to do for the whole day. Table 1 shows the performance of the 
brute force scheduling algorithm on a scenario shown in Table 1. Novel geo-task scheduling solutions that scale well in terms of the number of geo-tasks and the size of geographic areas are desirable.

Scheduling under various spatial and temporal constraints: Since the scheduling problem for mobile crowdsourcing is NPhard [2], heuristic-based algorithms [2] were proposed to support mobile crowdsourcing applications. However, a preferred scheduling solution should be able to take into account various spatiotemporal characteristics of real-world mobile crowdsourcing applications.

Task execution duration in mobile crowdsourcing applications: The existing scheduling schemes for mobile crowdsourcing [2] [7] fail to consider task execution duration. However, in reality it always requires a certain amount of time for a worker to accomplish a task. For example, in Gigwalk, a hotel gig may take a Gigwalker one to two hours to get it done [4] and the time to drive to the hotel could be a few minutes. Therefore, new spatiotemporal task scheduling solutions which incorporate the task execution duration are also desired.

Our goal and contributions: Given the location of a worker and the spatial distribution of tasks, the goal of our study is to provide scalable solutions in support of the near-optimal schedule (i.e., task execution sequence) that maximizes the number of the completed tasks by taking into account various spatial and temporal constraints, including the task execution duration and task expiration time. The contributions of this paper are summarized as follows:

- We formulate the spatiotemporal crowdsourcing problem that maximizes the number of the completed tasks by taking into account various spatial and temporal constraints, including the task execution duration and task expiration time.

- Due to the fact that the maximal task scheduling problem is NP-hard [2], we present two approximate solutions, LCPF and NUD-IC. LCPF solves the problem by incorporating particle filtering while NUD-IC generates the sub-optimal schedule by integrating particle filtering with DBSCAN clustering based on the concept of Non-Urgency Degree (NUD).

The remainder of this paper is organized as follows. Section 2 formulates the studied problem. In Section 3, we elaborate on LCPF and NUD-IC. Section 4 concludes the paper and presents our future work.

\section{PROBLEM FORMULATION}

Definition 2.1. $s$ is a spatial task located with three innate attributes: its location $s$, its execution duration $u_{s}$ and expiration time $e_{s}$.

In mobile crowdsourcing, each task will be located at a certain position in a spatial region and can only be accomplished there. Moreover, each task also has its own execution duration, which indicates how long it takes to complete the task, and expiration time, which indicates when the task is going to expire if it is still left unfinished. In this paper, we use an imaginary task $s_{0}$ to represent the spot where the worker is located originally. Similarly, $t_{0}$ represents the time when the worker starts from $s_{0}$, or the completion time of the imaginary task $s_{0}$.

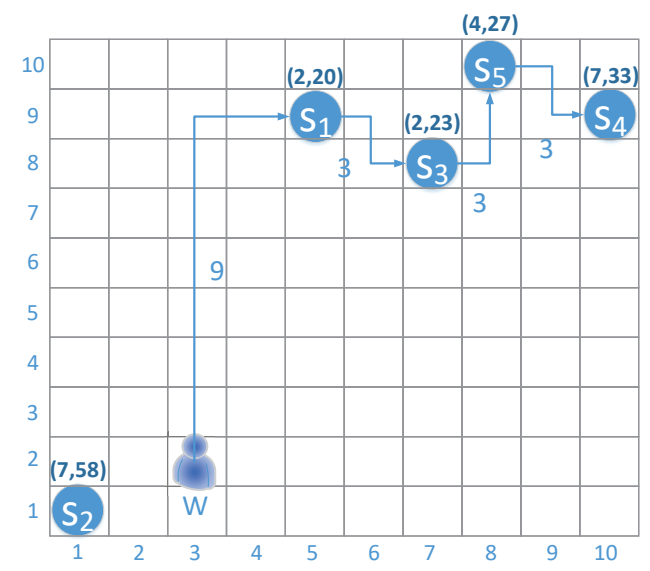

Figure 2: Parenthesized number pairs represent execution duration and expiration time with the same time unit as the scales in the grid.

Definition 2.2. A task set $S=\left\{s_{1}, s_{2}, \cdots, s_{n}\right\}$ is a collection of spatial tasks waiting to be accomplished, in which each task can be accomplished once and only once.

In our model, we assume a task set $S$ shares no task with any other set so that each worker is responsible for her own task set exclusively. Although in real-world cases, more than one worker may contend for the same tasks, it needs some special methods like competition, bargaining, collaboration and so on to cope with it, and these are not our major concerns in this paper. Therefore, those cases will be omitted here. We also assume that the worker will lose no time to head for the next one once the current task has been finished.

Definition 2.3. A task sequence $R_{\text {final }}=\left\{s_{r_{1}}, s_{r_{2}}, \cdots, s_{r_{m}}\right\}$ $\left(1 \leq r_{k} \leq n, 1 \leq k \leq m, 1 \leq m \leq n\right)$ is a succession of tasks assigned to or chosen by a worker from the whole task set $S$ such that all tasks in $R_{\text {final }}$ are distinct from each other (if $i \neq j$, then $\left.r_{i} \neq r_{j}, 1 \leq i, j \leq m\right)$ and are able to be accomplished before their respective expiration time. The number of elements in $R_{\text {final }}(m$ in this case) is defined as the length of task sequence.

Definition 2.4. The Geo-Task Scheduling (GTS) is the problem to find the longest task sequence obtained from a task set $S$, given the time when the worker starts from her original location.

Let's take the scenario shown in Figure 2 as an example. In Figure 2, distances are calculated in taxicab geometry (Manhattan length) and worker $w$ starts from $w$ at time 0 . If $w$ chooses $s_{2}$ as her first task to do, she will end up with only $s_{2}$ accomplished because the expiration time of all the other tasks does not leave her enough time to perform any of them at the completion time of $s_{2}$; however, if $w$ chooses $s_{1}$ as her first stop, she is going to end up with the longest task sequence $s_{1} \rightarrow s_{3} \rightarrow s_{5} \rightarrow s_{4}$. Table 2 summarizes the notations used in the problem formulation and the following algorithms. 
Table 2: Symbolic Notations

\begin{tabular}{ll}
\hline Symbol & Meaning \\
\hline$S$ & the whole task set \\
$e_{s}$ & the expiration time of task $s$ \\
$u_{s}$ & the execution duration of task $s$ \\
$s_{0}$ & an imaginary task representing where the worker starts \\
$t_{0}$ & the completion time of $s_{0}$ \\
$p t c l_{-} n o$ & number of samples in particle filtering \\
$R_{i}$ & a task sequence sample \\
$d d r$ & the number of groups to divide $S$ into \\
\hline
\end{tabular}

\section{ALGORITHM DESIGN}

In this section, we present two approximate solutions to solve the GTS problem.

\subsection{The Least Cost Neighbor with Particle Filtering (LCPF)}

LCPF is a particle filtering algorithm based on the heuristic of nearest neighbor. We present the definition of least cost neighbor based on nearest neighbor as below:

Definition 3.1. Given a task $s \in S$ accomplished at time $t_{s}$, let $S_{s u b}=\left\{s_{i} \mid t_{s}+\operatorname{dist}_{s, s_{i}}+u_{s_{i}} \leq e_{s_{i}}, s_{i} \in S, s \neq s_{i}\right\}$; if there exists a task $s^{*} \in S_{s u b}$ such that $\operatorname{dist}_{s, s^{*}}+u_{s^{*}} \leq \operatorname{dist}_{s, s_{i}}+u_{s_{i}}$ for any $s_{i} \in S_{s u b}$, then $s^{*}$ is the least cost neighbor (LCNB) of $s$ in $S$ at time $t_{s}$.

Searching for LCNBs of a task $s$ from the whole task set $S$ is actually to seek the nearest neighbors (considering the execution duration of tasks into the calculation of nearness) of $s$ in $S$, excluding tasks unable to be completed in time.

LCPF is detailed in Algorithm 1. First we get ptcl_no LCNBs of imaginary task $s_{0}$ (representing $w$ 's starting spot) from the whole task set $S$, each of which constitutes a task sequence, and put all the task sequences thus created into a queue $Q$, making sure $p t c l \_n o$ will not be greater than the number of tasks in $S$. After computing the completion time of each task sequence, which is when the last one in each task sequence finishes, we sort all the task sequences in the non-decreasing order of their completion time, i.e., the sooner a task sequence is finished the higher importance weight for that sequence. Then for each task sequence, we choose ptcl_no LCNBs, and append each of these ptcl_no tasks to the original sequence to generate ptcl_no new sequences with one of them replacing the original one which generated them. If not a single LCNB can be found, this sequence can be discarded. At last, we sort all the newly generated sequences thus (at most $p t c l_{-} n o^{2}$ ) in the non-decreasing order of their completion time and only keep the top ptcl_no ones and repeat the whole process until no more new task sequences can be generated. The last task sequence dropped from $Q$ will be chosen as the final result.

\subsection{Non-Urgency Degree Particle Filtering with Iterative Clustering (NUD-IC)}

In this subsection, we elaborate on the NUD-IC algorithm, which generates the sub-optimal schedule based on particle filtering, DBSCAN clustering, and the concept of Non-Urgency Degree (NUD) that is about to be introduced here. The basic idea behind the NUDIC algorithm is that if the completion of a task can leave more time for the accomplishment of other tasks before they expire, this task should be selected as the worker's next stop with a higher probability.

Given a task $s$ and its completion time $t_{s}$, the non-urgency degree (NUD) of $s$ for each task $s_{i} \in S$ at time $t_{s}$ can be defined as the following:

$$
N U D\left(s_{i}, s, t_{s}\right)=\left\{\begin{array}{l}
0: e_{s_{i}}-u_{s_{i}}-d i s t_{s, s_{i}}-t_{s} \leq 0 \\
\frac{e_{s_{i}}-u_{s_{i}}-d i s t_{s, s_{i}}-t_{s}}{u_{s_{i}}}: \text { otherwise }
\end{array}\right.
$$

NUD is a measurement of how early $s_{i}$ could be finished before its expiration comparing to its duration when the worker starts from $s$ at time $t_{s}$.

Similarly, for a given task sequence sample $R_{i}$ ended with task $s_{\text {end }}$ at time $t_{\text {end }}$, its sum of NUDs for $S$ can be defined as follows:

$$
N U D \_S U M\left(S, R_{i}\right)=\sum_{s_{i} \in S \backslash R_{i}} N U D\left(s_{i}, s_{\text {end }}, t_{\text {end }}\right)
$$

Based on Equation 1 and 2, we present particle filtering algorithm NUDPF, which serves as a subroutine in our NUD-IC algorithm. NUDPF is very similar to LCPF, except it uses Equation 2 for weight calculation and normalization instead of the completion time used in LCPF, and the importance weight of a task sequence sample $R_{i}$ is in proportion to the value of $\operatorname{NUD} \_\operatorname{SUM}\left(S, R_{i}\right)$. The details of NUDPF is omitted here.

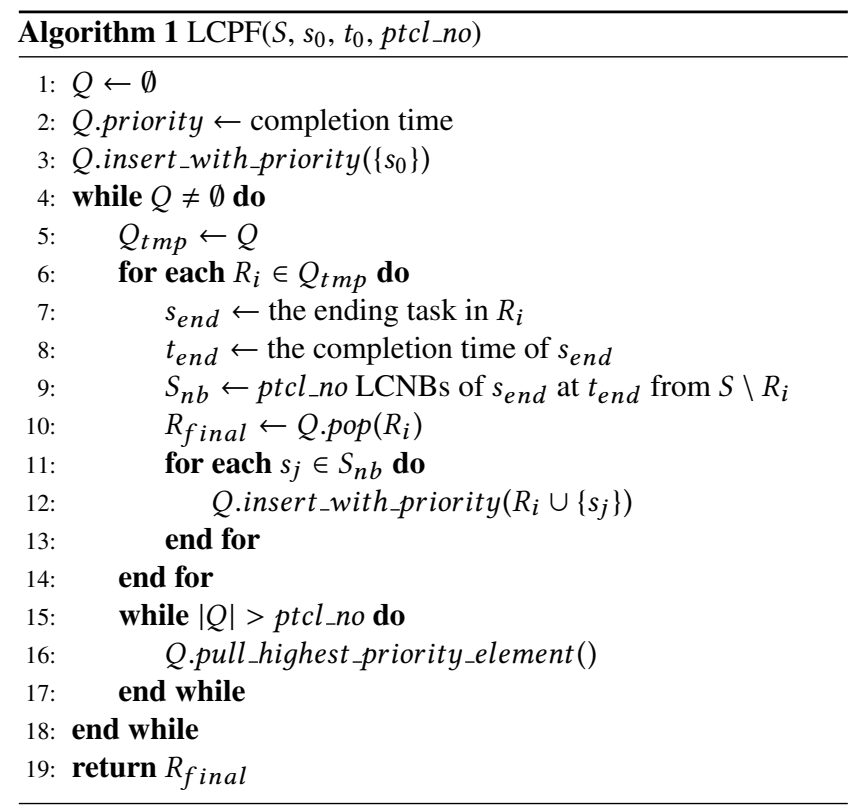

DBSCAN [3] is one of the most widely used data mining algorithms for clustering noisy data with outliers. However, it takes no temporal factor into account. We observe that tasks with long execution duration could be as unpromising as remote tasks. In our design, POIs are clustered via DBSCAN algorithm not only based on their relative distances to each other but also based on the homogeneity of their execution duration, which is called iterative clustering. Iterative clustering contains two steps: the first step is called duration categorization, which divides the whole task set into several groups according to each task's execution duration; the second step then applies DBSCAN algorithm to each group thus 
generated in turn to create clusters. This design guarantees that tasks with diverse execution duration will never be assigned into the same cluster no matter how close they are located geographically. To achieve this goal, besides the two traditional DBSCAN parameters, $\epsilon$ and MinPts, we introduce a third parameter duration division ratio $(d d r)$ into our iterative clustering process. Specifically, $d d r$ determines how many task groups $S$ should be divided into according to each task's execution duration.

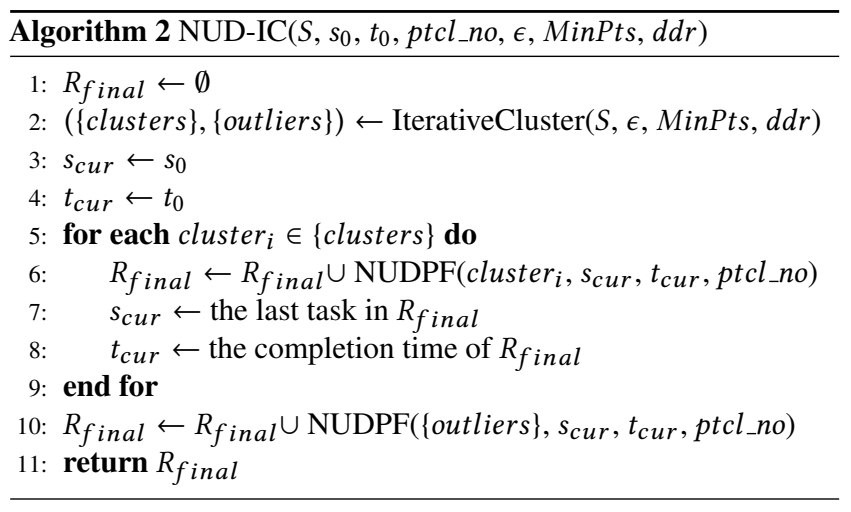

In duration categorization, we first pick up the minimum $u_{\text {min }}$ and maximum $u_{\max }$ out of the set $\left\{u_{s_{i}} \mid s_{i} \in S\right\}$ and create an interval $I=\left[u_{\min }, u_{\max }\right]$; second, we figure out the number of subintervals $\left(n_{d i v}\right)$ that the interval $I$ can be divided into via $n_{d i v}=$ $\lceil 1 / d d r\rceil$; third, letting $\Delta u=\left(u_{\max }-u_{\min }\right) * d d r$, we divide $I$ into $n_{\text {div }}$ subintervals: $I_{1}=\left[u_{\text {min }}, u_{\text {min }}+\Delta u\right], I_{2}=\left(u_{\text {min }}+\Delta u, u_{\text {min }}+\right.$ $2 * \Delta u], \ldots, I_{n_{d i v}-1}=\left(u_{m i n}+\left(n_{d i v}-2\right) * \Delta u, u_{\min }+\left(n_{d i v}-1\right) * \Delta u\right]$, $I_{n_{\text {div }}}=\left(u_{\min }+\left(n_{\text {div }}-1\right) * \Delta u, u_{\max }\right]$; lastly, we put tasks with their respective execution duration falling into the same subinterval $I_{i}$ into the same task group $G_{i}\left(1 \leq i \leq n_{d i v}\right)$, and thus divide the whole task set $S$ into $n_{d i v}$ task groups $G_{1}, \ldots, G_{n_{d i v}}$.

After the whole task set $S$ has been divided into $n_{d i v}$ task groups, $G_{1}, \ldots, G_{n_{d i v}}$ by the duration categorization process aforementioned, the first group $G_{1}$ containing tasks with the shortest execution duration will be processed by DBSCAN algorithm and the outliers thus extracted from $G_{1}$ will be added into $G_{2}$, which will be processed with DBSCAN algorithm too with its outliers being added into $G_{3}$ and so forth. When the last task group $G_{n_{d i v}}$ has been processed by DBSCAN algorithm, the outliers generated in this step comprise tasks with long execution duration and/or located geographically in some remote areas. Iterative clustering serves as the second subroutine in our NUD-IC algorithm. The detailed algorithm of iterative clustering is omitted here.

The algorithmic description and the block diagram of the whole Non-Urgency Degree Particle Filtering with Iterative Clustering (NUD-IC) are shown in Algorithm 2 and Figure 3, respectively. NUD-IC first divides the whole task set into clusters and a outlier set through iterative clustering, and then applies the aforementioned particle filtering method NUDPF to each cluster and the outlier set following the same order as how they are generated.

\section{CONCLUSION AND FUTURE WORK}

In this paper, in support of real-world spatiotemporal crowdsourcing applications, we study on the geo-task scheduling problem under

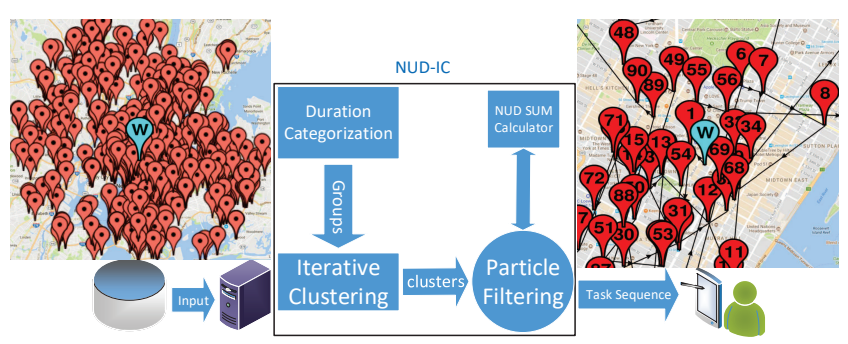

Figure 3: The block diagram of a crowdsourcing system utilizing NUD-IC algorithm.

various spatial and temporal constraints, including task execution duration and task expiration time. Given the location of a worker, the goal of our study is to find an optimal task execution sequence that maximizes the number of tasks that can be finished. We propose two scalable sub-optimal approaches, LCPF and NUD-IC, by leveraging particle filtering and DBSCAN clustering. In the future, we will incorporate road networks into our model to support road network-based geo-task scheduling for spatiotemporal crowdsourcing. Another task on our agenda is to extend our solutions to support task scheduling for multiple workers to maximize the revenue for group workers.

\section{ACKNOWLEDGMENTS}

This research has been funded in part by the National Science Foundation grants IIS-1618669 (III) and ACI-1642133 (CICI).

\section{REFERENCES}

[1] N. Carter. TaskRabbit: From Start-up to Global Web Market for Odd Jobs. http://www.inc.com/nicole-carter-and-tim-rice/taskrabbit-leah-busque-start-up-to-global-web-marketplacejobs.html, MAY 2012.

[2] D. Deng, C. Shahabi, U. Demiryurek, and L. Zhu. Task selection in spatial crowdsourcing from workers perspective. GeoInformatica, 20(3):529-568, 2016.

[3] M. Ester, H. Kriegel, J. Sander, and X. Xu. A Density-Based Algorithm for Discovering Clusters in Large Spatial Databases with Noise. In KDD, pages 226-231, 1996.

[4] I. Fried. Gigwalk Allows iPhone Owners to Stumble Into PartTime Work. http://allthingsd.com/20110504/gigwalk-allowsiphone-owners-to-stumble-into-part-time-work/, MAY 2011.

[5] E. Hamburger. How To Make Some Extra Cash Just By Taking Photos Around Town With Your iPhone. http://www.businessinsider.com/gigwalk-iphone-app-2011-5, May 2011.

[6] M. Hughes. What Is Uber And Why Is It Threatening Traditional Taxi Services? http://www.makeuseof.com/tag/uberthreatening-traditional-taxi-services/, July 2014.

[7] L. Kazemi and C. Shahabi. GeoCrowd: Enabling Query Answering with Spatial Crowdsourcing. In SIGSPATIAL, pages 189-198, 2012.

[8] S. H. Kim, Y. Lu, G. Constantinou, C. Shahabi, G. Wang, and R. Zimmermann. MediaQ: Mobile Multimedia Management System. In MMSys, pages 224-235, 2014. 